\title{
Multiwalled Carbon Nanotubes with Chemically Grafted Polyetherimides
}

Jason J. Ge, ${ }^{1}$ Dong Zhang, ${ }^{1}$ Qing Li,,${ }^{1}$ Haoqing Hou,${ }^{2}$ Matthew J. Graham, ${ }^{1}$

$$
\text { Liming Dai, }{ }^{3} \text { Frank W. Harris, }{ }^{1} \text { and Stephen Z. D. Cheng }{ }^{1 *}
$$

${ }^{I}$ Maurice Morton Institute and Department of Polymer Science, The University of Akron, Akron, $\mathrm{OH} 44325, \mathrm{USA} ;{ }^{2}$ Chemistry College of Jiangxi Normal University, Nanchang, JX 330027, China; ${ }^{3}$ Department of Chemical and Materials Engineering, University of Dayton, Dayton, $\mathrm{OH} 45469$, USA

\section{Gel Permeation Chromatography (GPC) Measurements}

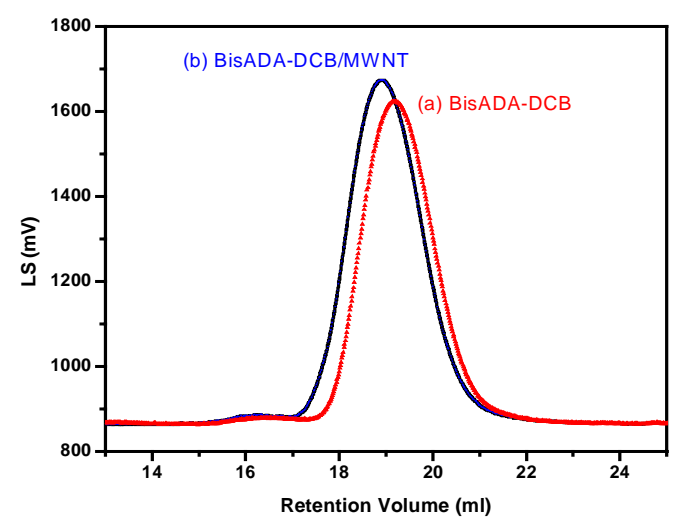

Figure S1. GPC results of (a) the neat polyetherimide, and (b) the polyetherimide containing 0.14 wt. \% carboxylic acid-functionalized MWNTs

The molecular weight and molecular weight distribution of the completely imidized pure BisADA-DCB and BisADA-DCB- $g$-MWNTs ( 0.1 wt. \% MWNTs) were determined by Gel Permeation Chromatography (GPC), coupled with a $90^{\circ}$ laser light scattering detector (Wyatt Dawn EOS), a viscometer detector (Viscoteck Model 100) and a reflectometer detector (Waters 410). The average molecular weights were measured in THF at $35^{\circ} \mathrm{C}$ through a $0.2 u \mathrm{~m}$ PTFE filter with a polystyrene standard calibration. The pure BisADA-DCB possessed a number average molecular weight $\left(M_{n}\right)$ of $8390 \mathrm{~g} / \mathrm{mol}$ with a polydispersity (PDI) of 2.13 (Figure S1a). 
The $M_{n}$ of the BisADA-DCB- $g$-MWNTs $(0.1$ wt. \%) slightly increased to $9900 \mathrm{~g} / \mathrm{mole}$ (Figure S1b) with a PDI of 2.09 .

\section{TGA Experimental Results}

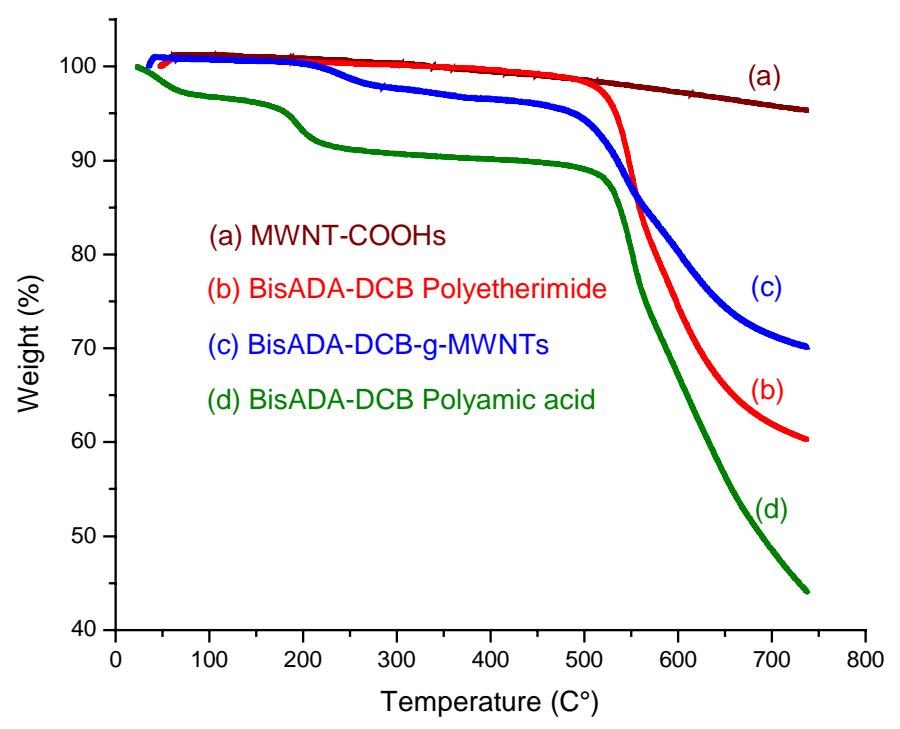

Figure S2. TGA plots for (a) the acid-functionalized MWNTs; (b) the neat BisADA-DCB polyetherimide; (c) the BisADA-DCB- $g$-MWNTs (10/4); and (d) the neat BisADA-DCB polyamic acid

The thermal stability and thermal decomposition temperature of completely imidized pure BisADA-DCB, acid-functionalized MWNTs and BisADA-DCB- $g$-MWNTs were measured up to $740{ }^{\circ} \mathrm{C}$ at a heating rate of $10{ }^{\circ} \mathrm{C} / \mathrm{min}$ in a $\mathrm{N}_{2}$ atmosphere using thermogravimetric analysis (TGA, TA 2050). The weight loss for the acid-functionalized MWNTs is about $5 \%$ at $735^{\circ} \mathrm{C}$ in Figure S2a. A 5\% weight loss of the fully imidized BisADA-DCB containing amine end groups is reached at $535^{\circ} \mathrm{C}$, as shown in Figure S2b. The residual weight at $735^{\circ} \mathrm{C}$ is about $60 \%$.

We also designed an experiment to monitor the further imidization of the BisADA-DCB- $g$ MWNTs at a weight ratio of 10 to 4 . The purpose of generating this high weight ratio is due to an enhancement of the experimental signals. After the fully imidized pure BisADA-DCB with amine end groups was mixed with the acid-functionalized MWNTs, the sample was dried. It was 
then heated to $150-160{ }^{\circ} \mathrm{C}$ for 96 hours in the solid state to graft the BisADA-DCB onto the MWNTs before the TGA experiment was conducted. Figure S2c shows the results which exhibit a new weight loss at an onset temperature of $\sim 160{ }^{\circ} \mathrm{C}$ upon heating. This additional weight loss could be due to a new imidization reaction between the BisADA-DCB with amine end groups and acid-functionalized MWNTs, which results in the removal of $\mathrm{H}_{2} \mathrm{O}$. In order to confirm this imidization process, a controlled BisADA-DCB polyamic acid was synthesized. The TGA results of this polyamic acid sample in Figure S2d show a similar weight loss at an onset temperature of $\sim 140^{\circ} \mathrm{C}$ due to the thermal imidization of the BisADA-DCB polyamic acid in TGA. It is thus evident that the formation of the imide rings between the BisADA-DCB with amine end groups and the acid-functionalized MWNTs occurs at elevated temperature. This is consistent with our explanation of the ATR results (Figure 1 in the communication).

\section{UV/Vis Experimental Results}

The UV/Vis absorption spectra of the acid-functionalized MWNTs, pure BisADA-DCB, and the BisADA-DCB-g-MWNTs were measured from 190 to $600 \mathrm{~nm}$ using an HP 8453 photospectrometer. The absorption peak of the MWNTs in THF is at about $255 \mathrm{~nm}$ in Figure 3 of the communication. Before grafting, the pure polyimide possesses two major peaks at 225 and $265 \mathrm{~nm}$ with a broad tail from 310 to $360 \mathrm{~nm}$ in Figure S3b. After the attachment, the BisADADCB- $g$-MWNTs possesses two major peaks at 230 and $280 \mathrm{~nm}$ with a broad shoulder from 325 to $370 \mathrm{~nm}$ as shown in Figure 3 of the communication. Interestingly, a red shift in the absorption has been observed after the chemical attachment. This has not so far been addressed in polyimide-g-carbon nanotube systems. This observation suggests that the conjugated length of the grafted BisADA-DCB molecules increases when the molecules are chemically attached to the surfaces of the MWNTs. 


\section{Mechanical Testing Results}

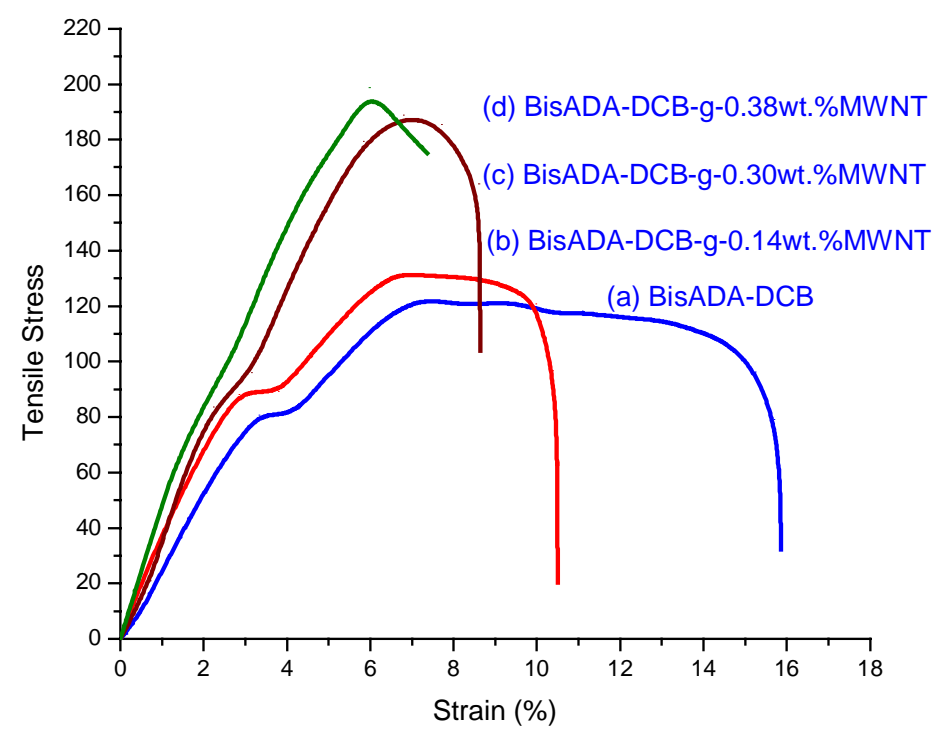

Figure S4. Relationships between tensile stresses and straines for the (a) pure BisADA-DCB film; (b) BisADA-DCB-g-MWNT (0.14 wt. \%) film; (c) BisADA-DCB- $g$-MWNT (0.25 wt. \%) film; and (d) BisADA-DCB- $g$-MWNT (0.38 wt. \%) film

In order to understand the improvement in mechanical properties with the loading of the oxidized MWNTs, the tensile strength and modulus were evaluated using an Instron (5543) tensile test at ambient temperature, with a gauge length of $5 \mathrm{~mm}$ and a crosshead speed of 2 $\mathrm{mm} / \mathrm{min}$. The specimens were cut from the solution cast films with a typical size of $20 \mathrm{~mm}$ (length) x $2 \mathrm{~mm}$ (width).

The tensile strength and modulus of the BisADA-DCB film grafted with $0.14,25,38$ wt. \% carboxylic acid-functionalized MWNTs increased to 131, 186, $194 \mathrm{MPa}$ and 3.7, 4.1, 4.4 GPa, respectively, with corresponding elongations at the break of $10 \%, 8 \%$, and $7 \%$. This is compared to $\sim 121 \mathrm{MPa}$ and $\sim 2.9 \mathrm{GPa}$ in the pure BisADA-DCB films with an elongation at the break of $16 \%$. With an increase in the loading of the small amount of the MWNTs, the composite films became stiffer and stronger due to chemical and physical interactions between the BisADA- 
DCB-g-MWNTs and the amine-terminated BisADA-DCB molecules. In addition to the 1D structured filler effect, the large quantity of the chemical grafted interfaces between the MWNTs and the BisADA-DCB molecules could also be important in improving the mechanical performance. 